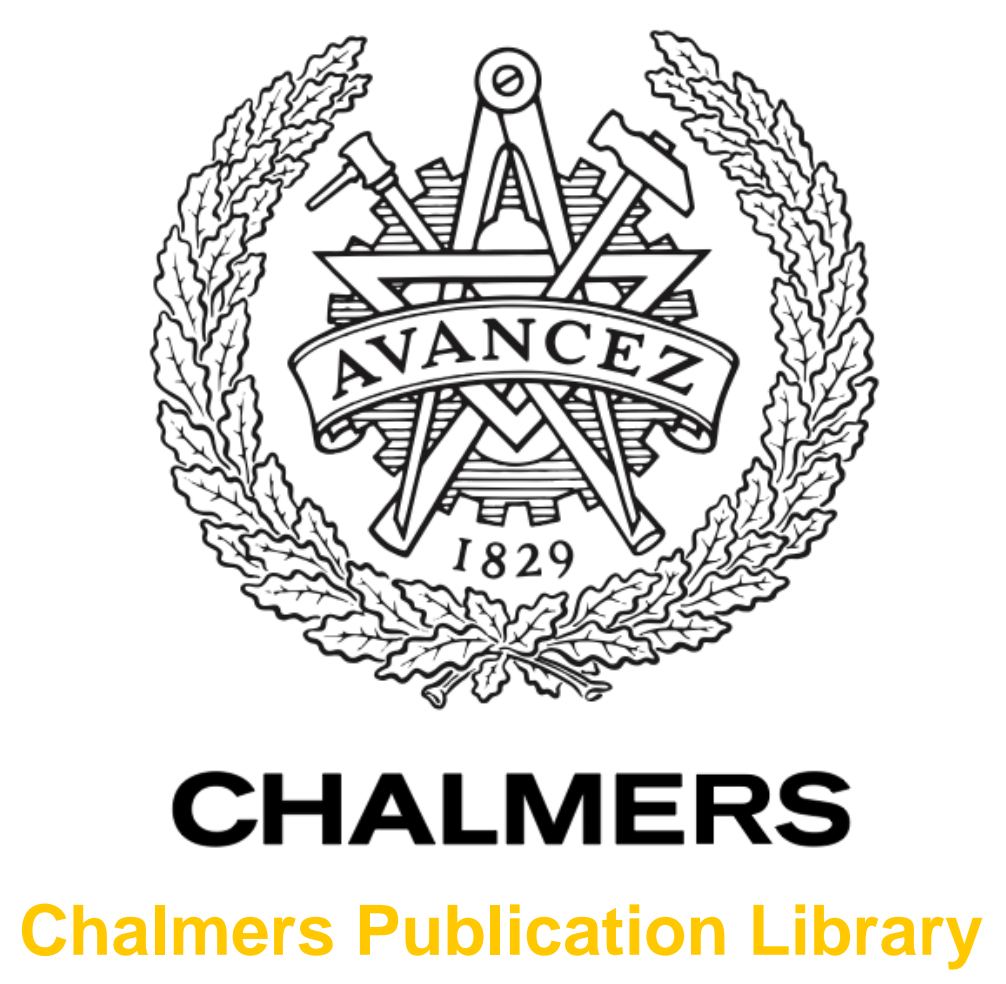

\title{
Scattering of a SH-wave by an elastic fiber of nonclassical cross section with an interface crack
}

This document has been downloaded from Chalmers Publication Library (CPL). It is the author's version of a work that was accepted for publication in:

Mechanics of composite materials (ISSN: 0191-5665)

Citation for the published paper:

Kunets, Y.; Matus, V. ; Mykhavskiv, V. et al. (2008) "Scattering of a SH-wave by an elastic fiber of nonclassical cross section with an interface crack". Mechanics of composite materials, vol. 44(2), pp. 165-172.

http://dx.doi.org/10.1007/s11029-008-9002-4

Downloaded from: http://publications.lib.chalmers.se/publication/73880

Notice: Changes introduced as a result of publishing processes such as copy-editing and formatting may not be reflected in this document. For a definitive version of this work, please refer to the published source. Please note that access to the published version might require a subscription. 


\title{
Scattering of SH-wave by an elastic fiber of non-classical cross-section with interface crack
}

\author{
Yaroslav Kunets ${ }^{\mathrm{a}}$, Valerij Matus a , Victor Mykhas'kivª, \\ Anders Boström ${ }^{\mathrm{b}}$, Chuanzeng Zhang ${ }^{\mathrm{c}}$ \\ ${ }^{a}$ Pidstryhach Institute for Applied Problems of Mechanics and Mathematics, Lviv 79060, Ukraine \\ ${ }^{\mathrm{b}}$ Department of Applied Mechanics, Chalmers University of Technology, Goteborg 41296, Sweden \\ ${ }^{\mathrm{c}}$ Department of Civil Engineering, University of Siegen, Siegen 57068, Germany
}

\begin{abstract}
The problem of interaction of a plane time-harmonic SH-wave with an elastic inclusion of quasi-square and quasi-triangular cross-sections, when an interface crack is present between the infinite elastic matrix and the fiber, is considered. The modified null field method taking into account the asymptotic behavior of the solution at the crack tips is exploited for obtaining the numerical results. The effects of fiber shape, inclusion/matrix materials combination, debonding (crack size) and direction of wave incidence on the scattering amplitude in the far zone are analyzed.
\end{abstract}

Key words: Elastodynamic scattering; Elastic inclusion of non-classical shape; Interface crack; Null field approach

\section{Introduction}

In many cases the degradation of physical properties of fiber-reinforced composites is caused by cracks at the interfaces. Partial debonding can have a strong influence on the field pattern of scattered waves. This fact is important from the point of view of nondestructive evaluation of composite materials by ultrasonic methods, experimental data interpretation, etc.

Investigations of wave scattering by an interface crack between a matrix and a cylindrical or elliptical fiber have been performed by Norris and Yang [1], Yang and Norris [2], Zhang and Gross [3], Wang and Wang [4], and Sato and Shindo [5], involving the basic formulation of boundary integral equation methods for elastodynamic inclusion problems (see Kitahara et al. [6]). The crack opening displacements across the crack faces and the contact stresses across the bonded fraction of the interface are the unknown functions in the equations obtained, which are solved numerically. The analysis was extended to the effective dynamic properties of cracked composites with distributed inclusions of such types by Zhang and Gross [3, 7], Gross and Zhang [8], and Sato and Shindo [5]. But fibers of more complicated cross-sections were not considered.

In this paper elastic inclusions of quasi-square and quasi-triangular cross-sections (with smoothed edges) and interface cracks subjected to incident plane time-harmonic elastic SH-waves are included into the analysis. The null field (T-matrix) method is applied for the solution of the scattering problem. The method was originally developed for acoustic scattering by Waterman [9], and has later been extended to the scattering of waves in elastic solids by voids, perfectly bonded rigid and elastic 
inclusions, inclusions with thin interface layers by Varatharajulu and Pao [10], Olsson and Boström [11], Boström et al. [12], Emets et al. [13]. Longer lists of references on the subject can be found in the review works by Martin [14], Beskos [15], and Boström [16]. The transition matrices and solutions for problems of wave propagation in an infinite elastic homogeneous solid with a nonplanar crack were proposed by Boström and Olsson [17]. The "square-root" behavior of the solution at the crack edge is explicitly considered in that paper. In the present paper the idea is realized for an interface crack in a fiber-reinforced composite under antiplane shear conditions, when unknown displacements and stresses at the interface are expressed as infinite series trigonometric functions. Such an approximation increases considerably the accuracy of calculation of the scattering parameters. By using these expansions for the unknowns in the moment equations that follow from the outer and inner integral representations and by applying the continuity and the traction-free conditions on the bonded and debonded interfaces, a system of linear algebraic equations for the expansion coefficients is constructed. On this base the scattering amplitudes in the far field for the different fiber shapes, inclusion/matrix materials combinations, crack sizes and directions of wave incidence are studied.

\section{Scattering problem and null field method implementation}

Consider an elastic unbounded medium with shear modulus $\mu_{1}$ and density $\rho_{1}$ where there is an inclusion of corresponding parameter $\mu_{2}, \rho_{2}$, which occupies the region $W_{2}$. There is an interface crack on a part of the material interface $S_{0}$ and the conditions of ideal contact are fulfilled on the line $S_{1}=\partial W_{2} \backslash S_{0}$ (see Fig. 1). Time-harmonic conditions are assumed and only antiplane conditions are considered, therefore the total displacements both in the matrix and inclusion $u^{1}(\mathbf{x}), u^{2}(\mathbf{x})$, respectively, satisfy the Helmholtz equation $\left(W_{1}=R^{2} \backslash W_{2}\right)$

$$
\left(\Delta+k_{j}^{2}\right) u^{j}(\mathbf{x})=0, \quad \mathbf{x} \in W_{j}, \quad j=1,2,
$$

and the following boundary conditions:

$$
\begin{array}{ll}
u^{2}(\mathbf{x})=u^{1}(\mathbf{x}), & \frac{\partial u^{2}(\mathbf{x})}{\partial n}=\gamma \frac{\partial u^{1}(\mathbf{x})}{\partial n}, \quad \gamma=\mu_{1} / \mu_{2}, \quad \mathbf{x} \in S_{1} \\
\frac{\partial u^{j}(\mathbf{x})}{\partial n}=0, & j=1,2, \quad \mathbf{x} \in S_{0} .
\end{array}
$$


Here $\mathbf{x}=\left(x_{1}, x_{2}\right)$ are Cartesian coordinates; $\mathbf{n}$ is the outer normal direction to the $\partial W_{2} ; k_{1}$ and $k_{2}$ are the transverse wave numbers in the matrix and in the inclusion, respectively. A time factor $\exp (-i \omega t)(\omega$ is the angular frequency) is assumed and hereafter suppressed.

We introduce the scattered field $u^{s}(\mathbf{x})$ as the difference between the total field and the incident one in $W_{1}$

$$
u^{s}(\mathbf{x})=u^{1}(\mathbf{x})-u^{i n}(\mathbf{x})
$$

It is required to satisfy the Sommerfeld radiation condition at infinity (see Achenbach [18]), from which it follows that:

$$
u^{s}(\mathbf{x})=\frac{e^{i k_{1} r+i \pi / 4}}{\sqrt{8 \pi k_{1} r}} f(\omega, \theta), \quad r \rightarrow \infty, \quad \mathbf{x}=(r \cos \theta, r \sin \theta)
$$

Here $f(\omega, \theta)$ is the complex amplitude or far-field pattern of the scattered wave, $(r, \theta)$ are polar coordinates.

Henceforth we shall assume that a plane horizontally polarized transverse wave is incident on the inclusion

$$
\begin{aligned}
& u^{i n}(\mathbf{x})=u_{0} \exp \left[-i k_{1}\left(x_{1} \cos \theta_{i n}+x_{2} \sin \theta_{i n}\right)\right]=u_{0} \sum_{\sigma, m} \varepsilon_{m} b_{\sigma m}^{i n} \operatorname{Re} \psi_{\sigma m}^{1}(\mathbf{x}), \\
& \psi_{\sigma m}^{j}(\mathbf{x})=H_{m}^{(1)}\left(k_{j} r\right) C_{\sigma m}(\theta), \quad j=1,2, \quad C_{\sigma m}(\theta)= \begin{cases}\cos m \theta, & \sigma=1, m=0,1, \ldots, \\
\sin m \theta, & \sigma=2, m=1,2, \ldots,\end{cases} \\
& b_{\sigma m}^{i n}=(-i)^{m} C_{\sigma m}\left(\theta_{i n}\right) .
\end{aligned}
$$

Here $H_{m}^{(1)}(x)$ are the Hankel functions of the first kind, $\theta_{i n}$ is the angle of incidence and $u_{0}$ is the amplitude of the wave, $\varepsilon_{m}=2-\delta_{m 0}\left(\delta_{m 0}\right.$ is the Kronecker delta). The basis fuctions with "Re" in front contain a Bessel function instead of a Hankel function.

With the purpose of finding a numerical solution to the exterior mixed problem (1) - (3) inhe far-field zone of a scatterer we use the null field approach. Then, from the outer and inner integral representations we can derive

$$
\int_{S}\left[u^{1} \frac{\partial}{\partial n} \psi_{\sigma m}^{1}-\psi_{\sigma m}^{1} \frac{\partial}{\partial n} u^{1}\right] d S=4 i u_{0} b_{\sigma m}^{i n}
$$




$$
\int_{S}\left[u^{2} \frac{\partial}{\partial n} \operatorname{Re} \psi_{\sigma m}^{2}-\left(\operatorname{Re} \psi_{\sigma m}^{2}\right) \frac{\partial}{\partial n} u^{2}\right] d S=0, \quad S=\partial W_{2}
$$

The representation of the unknown functions on $S$ can be realized in different ways. Here we use the modified null field approach developed by Boström and Olsson [17], in which the behavior of the solution at the points of changing boundary conditions on $S$ is accounted for explicitly. The asymptotic behavior for displacements and stresses can be determined by the technique proposed by Sinclair [19]. Therefore, the unknown surface fields are expanded in the following way:

$$
\begin{aligned}
& v(\theta)=\sum_{\sigma, m} x_{\sigma m}^{1} C_{\sigma m}(\theta), \quad u^{2}(\theta)=\sum_{\sigma, m} x_{\sigma m}^{2} C_{\sigma m}(\theta), \\
& v(\theta)= \begin{cases}a Z(\theta) \frac{\partial u^{1}(\theta)}{\partial n}, & \theta \in S_{1}, \\
Z(\theta)\left[u^{1}(\theta)-u^{2}(\theta)\right], \theta \in S_{0},\end{cases} \\
& Z(\theta)=\left\{\begin{array}{l}
2 \tau(\theta) \frac{\sqrt{\left(2 \pi+\theta_{0}-\theta\right)\left(\theta-\theta_{1}\right)}}{\sqrt{2 \pi+\theta_{0}-\theta_{1}}}, \\
(1+\gamma)^{-1} \tau^{-1}(\theta) \frac{\sqrt{\theta_{1}-\theta_{0}}}{\sqrt{\left(\theta-\theta_{0}\right)\left(\theta_{1}-\theta\right)}}, \theta \in S_{1},
\end{array}\right.
\end{aligned}
$$

where $v(\theta)$ and $u^{2}(\theta)$ are continuous over $S, a$ is the characteristic dimension of the inclusion, the given function $r(\theta)$ describes the contour of the fiber in the polar coordinates, $\theta_{0}$ and $\theta_{1}$ are the angle coordinates of the crack tips (Fig. 1).

Substituting these expansions into the equations (6) and (7) and using the boundary condition (2) and (3) we obtain an infinite system of linear algebraic equations for the unknown coefficients $x_{\sigma m}^{l}(l=1,2)$ :

$$
\begin{aligned}
& \sum_{\sigma^{\prime}, m^{\prime}}\left(a_{\sigma m, \sigma^{\prime} m^{\prime}}^{1} x_{\sigma^{\prime} m^{\prime}}^{1}+a_{\sigma m, \sigma^{\prime} m^{\prime}}^{2} x_{\sigma^{\prime} m^{\prime}}^{2}\right)=4 i u_{0} b_{\sigma m}^{i n}, \\
& \sum_{\sigma^{\prime}, m^{\prime}}\left(a_{\sigma m, \sigma^{\prime} m^{\prime}}^{3} x_{\sigma^{\prime} m^{\prime}}^{1}+a_{\sigma m, \sigma^{\prime} m^{\prime}}^{4} x_{\sigma^{\prime} m^{\prime}}^{2}\right)=0 \\
& a_{\sigma m, \sigma^{\prime} m^{\prime}}^{1}=\int_{S_{0}} Z^{-1}(\theta) C_{\sigma^{\prime} m^{\prime}}(\theta) \frac{\partial}{\partial n} \psi_{\sigma m}^{1} d S-a^{-1} \int_{S_{1}} Z^{-1}(\theta) C_{\sigma^{\prime} m^{\prime}}(\theta) \psi_{\sigma m}^{1} d S,
\end{aligned}
$$




$$
\begin{aligned}
& a_{\sigma m, \sigma^{\prime} m^{\prime}}^{2}=\int_{S} C_{\sigma^{\prime} m^{\prime}}(\theta) \frac{\partial}{\partial n} \psi_{\sigma m}^{1} d S, a_{\sigma m, \sigma^{\prime} m^{\prime}}^{3}=-\gamma a^{-1} \int_{S_{1}} Z^{-1}(\theta) C_{\sigma^{\prime} m^{\prime}}(\theta) \operatorname{Re} \psi_{\sigma m}^{2} d S, \\
& a_{\sigma m, \sigma^{\prime} m^{\prime}}^{4}=\int_{S} C_{\sigma^{\prime} m^{\prime}}(\theta) \frac{\partial}{\partial n} \operatorname{Re} \psi_{\sigma m}^{2} d S .
\end{aligned}
$$

The scattering amplitude, as follows from Eqs. (4) and (8) and the Helmholtz integral representation for $u^{s}(\mathbf{x})$, is directly connected with the coefficients $x_{\sigma m}^{l}(l=1,2)$ :

$$
\begin{aligned}
& f(\omega, \boldsymbol{v})=\sum_{\sigma, m} \varepsilon_{m} i^{-m} A_{\sigma m} C_{\sigma m}(\theta), \\
& A_{\sigma m}=\sum_{\sigma^{\prime}, m^{\prime}}\left(x_{\sigma^{\prime} m^{\prime}}^{1} \operatorname{Re} a_{\sigma m, \sigma^{\prime} m^{\prime}}^{1}+x_{\sigma^{\prime} m^{\prime}}^{2} \operatorname{Re} a_{\sigma m, \sigma^{\prime} m^{\prime}}^{2}\right) .
\end{aligned}
$$

Consequently, expression (10) gives the solution of the problem in the far wave zone of the scatterer.

\section{Numerical results}

As an illustration of the foregoing algorithm, we investigate scattering of a plane $\mathrm{SH}$-wave by fiber of nonclassical cross-section with an interface crack. We consider inclusions of quasi-square $(N=3)$ and quasi-triangular $(N=2)$ shapes, which are given by the relations

$$
\begin{aligned}
& r(\alpha)=\frac{a}{1+\varepsilon} \sqrt{1+\varepsilon^{2}-2 \varepsilon \cos (N+1) \alpha}, \quad \theta(\alpha)=\operatorname{arctg} \frac{\sin \alpha+\varepsilon \sin N \alpha}{\cos \alpha-\varepsilon \cos N \alpha}, \\
& 0 \leq \alpha \leq 2 \pi .
\end{aligned}
$$

Here the cross-sections of the inclusions are parametrized by $\varepsilon$ (see Fig. 2), so that the cylindrical inclusion has $\varepsilon=0$.

Calculation have been performed for three combinations of matrix and fiber: (i) glass/epoxy composite $\left(\mu_{1}=1.28 \mathrm{GPa}, \rho_{1}=1250 \mathrm{~kg} / \mathrm{m}^{3}, \mu_{2}=29.9 \mathrm{GPa}, \rho_{2}=2550 \mathrm{~kg} / \mathrm{m}^{3}\right)$; (ii) epoxy $/ \mathrm{glass}$ composite (inverse to (i)); (iii) glass/aluminium composite ( $\mu_{1}=26.49 \mathrm{GPa}, \rho_{1}=2700 \mathrm{~kg} / \mathrm{m}^{3}$, $\left.\mu_{2}=29.9 \mathrm{GPa}, \rho_{2}=2550 \mathrm{~kg} / \mathrm{m}^{3}\right)$.

It is obvious that in the numerical calculations the sums in $m$ in equations (9) and (10) have to be truncated. Otherwise the calculation of the quantities $x_{\sigma m}^{l}(l=1,2)$ can be calculated by standard 
numerical procedures. Indeed, in our analysis an acceptable accuracy is obtained, if the sums are truncated at $m=6+m_{d}$ for $k_{1} a \leq 3$ and $m=E\left(2 k_{1} a\right)+m_{d}$ for $3<k_{1} a \leq 10$, where $E(x)$ is the entire part of $x, a$ is the characteristic dimension of the inclusion (Fig. 2). The parameter $m_{d}$ is determined by numerical experiments.

In Figs 3-6 we show the dimensionless scattering amplitudes $f_{0}=u_{0}^{-1}\left|f\left(\omega, \theta_{s c}\right)\right|\left(\theta_{s c}\right.$ is the angle of observation) as a function of the normalized wave number $k_{1} a$ for different matrix/inclusion/interface crack constructions. Bold lines in Figs. 4-6 correspond to the case of a fully bonded inclusion.

The curves in the Fig. 3 depict the backscattering amplitudes, when $\theta_{i n}=\theta_{s c}=0^{\circ}$, for the different inclusion shapes. The crack is at the material interface in the glass/epoxy composite and has the tip coordinates $\theta_{1}=-\theta_{0}=45^{\circ}$ (quasi-square, Fig. 3a) and $\theta_{1}=-\theta_{0}=60^{\circ}$ (quasi-triangle, Fig. 3b). For the results presented in Fig. 3 the truncation parameter $m_{d}=6$ for the quasi-square inclusion and $m_{d}=9$ for the quasi-triangular inclusion was used. From the figure follows that the influence of the inclusion cross section on the backscattering amplitude is most expressed in the range of higher wave numbers, namely for $k_{1} a>4.0$ in the case of quasi-square shape and for $k_{1} a>3.0$ in the case of quasi-triangular shape. As the shape parameter $\varepsilon$ and wave number $k_{1} a$ become bigger the convergence of the results is getting slower. That is the reason the termination of the curve for $\varepsilon=0.25$ from $k_{1} a=5.0$ in Fig. $3 \mathrm{a}$ and for $\varepsilon=0.35$ from $k_{1} a=3.0$ in Fig $3 \mathrm{~b}$.

The response of the same composite under fixed quasi-square or quasi-triangular inclusion shape and crack size as above on the incoming wave is described for different angles of observation in Fig. 4. The presence of the crack causes significant deviations in the scattering amplitude for $\theta_{s c}=0^{\circ}$ and $\theta_{s c}=180^{\circ}$, unlike the scattering angle $\theta_{s c}=90^{\circ}$.

The effects of matrix/inclusion materials combination and size of interface crack on the backscattering amplitude are analyzed in the Fig. 5 and Fig. 6, respectively, for the quasi-square inclusion. The crack in the Fig. 5 has the tip coordinates $\theta_{1}=-\theta_{0}=45^{\circ}$, the variation of crack size in Fig. 6 is given by $\theta_{0}=-45^{\circ}, \theta_{1}=\theta_{0}+\theta_{*}$. The values $m_{d}=12$ for epoxy/glass composite and $m_{d}=3$ in other cases are the optimal values of the parameter $m_{d}$ in the calculations. As seen from the illustration in the case of inclusions of low rigidity (epoxy/glass composite) the frequency dependence of the amplitude $f_{0}$ is of resonance nature, which is not observed for other relations of the 
composite components' rigidities. For low contrast inclusions (glass/aluminium composite, $\gamma \approx 1$ ), an increase of the interface crack size causes an increase of the level of scattering amplitude in the far field, which is just to be expected.

Fig. 7 presents the graph of monostatic scattering $\left(\theta_{s c}=\theta_{i n}\right)$ for the glass/epoxy composite with the quasi-triangular inclusion. The fully bonded and a debonded $\left(\theta_{1}=-\theta_{0}=60^{\circ}\right)$ inclusion are considered (the lower and upper parts of the graph, respectively). These graphs show that when the frequency $k_{1} a$ increases WHAT DO YOU WANT TO SAY IN THE REST OF THE SENTENCE? the increase of amplitude of inverse scattering is observed in directions perpendicular to the inclusion sides.

\section{Acknowledgement}

This research was supported by INTAS under Project No. 05-1000008-7979.

\section{References}

[1] A.N. Norris, Y. Yang, Dynamic stress on a partially bonded fiber, ASME J. Appl. Mech. 58 (1991) 404-409.

[2] Y. Yang, A.N. Norris, Shear wave scattering from a debonded fiber, J. Mech. Phys. Solids 39 (1991) 273-274.

[3] Ch. Zhang, D. Gross, On Wave Propagation in Elastic Solids with Cracks, Computational Mechanics Publications, Southampton, 1998.

[4] Y-H. Wang, D. Wang, Scattering of elastic waves by a rigid cylindrical inclusion partially debonded from its surrounding matrix. I. SH case, Int. J. Solids Structures 33 (1996) 2789-2815.

[5] H. Sato, Y. Shindo, Influence of microstructure on scattering of plane elastic waves by a distribution of partially debonded elliptical inclusions, Mechanics of Materials 34 (2002) 401-409.

[6] M. Kitahara, K. Nakagawa, J.D. Achenbach, Boundary-integral equation method for elastodynamic scattering by a compact inhomogeneity, Computational Mechanics 5 (1989) 129-144.

[7] Ch. Zhang, D. Gross, Wave attenuation and dispersion in fiber-reinforced composites with interface shipping, in: Z.P. Bazant (Ed.), Fracture and Damage in Quasibrittle Structures, E \& FN Spon, 1994, pp. 633-640.

[8] D. Gross, Ch. Zhang, On wave propagation in two-phase materials with interface defects, in: G. Baker, B.L. Karihaloo (Eds.) Fracture of Brittle Disordered Materials: Concrete, Rock and Ceramics, E \& FN Spon, 1994.

[9] P.C. Waterman, New formulation of acoustic scattering, J. Acoust. Soc. Amer. 45 (1968) 1417-1429.

[10] V. Varatharajulu, Y.H. Pao, Scattering matrix of elastic wave, J. Acoust. Soc. Amer. 60 (1976) 1361-1371.

[11] P. Olsson, A. Boström, Elastic waves propagation in the presence of crack and thin interface layers, J. Tech. Phys. 31 (1990) 393-400. 
[12] A. Boström, P. Olsson, S.K. Datta, Effective plane wave propagation trough a medium with spheroidal inclusions surrounded by thin interface layers, Mechanics of Materials 14 (1992) 59-66.

[13] V.F. Emets, Ya.I. Kunets, V.V. Matus, Scattering of SH waves an elastic thin-walled rigidly supported inclusion, Arch. Appl. Mechanics 73 (2004) 769-780.

[14] P.A. Martin, On connections between boundary integral equations and T-matrix methods, Eng. Anal. Bound. Elem. 27 (2003) 771-777.

[15] D.E. Beskos, Boundary element methods in dynamic analysis: Part II (1986-1996), Appl. Mech. Rev. 50 (1997) 149197.

[16] A. Boström, Review of hypersingular integral equation method for crack scattering and application to modeling of ultrasonic nondestructive evaluation, Appl. Mech. Rev. 56 (2003) 383-405.

[17] A. Boström, P. Olsson, Scattering of elastic waves by non-planar cracks, Wave Motion 9 (1987) 61-76.

[18] J.D. Achenbach, Wave Propagation in Elastic Solids, North-Holland, Amsterdam, 1973.

[19] G.B. Sinclair, On the singular eigenfunctions for plane harmonic problems in composite regions, ASME. J. Appl. Mech. 47 (1980) 87-92. 
FIGURES AND FIGURE CAPTIONS

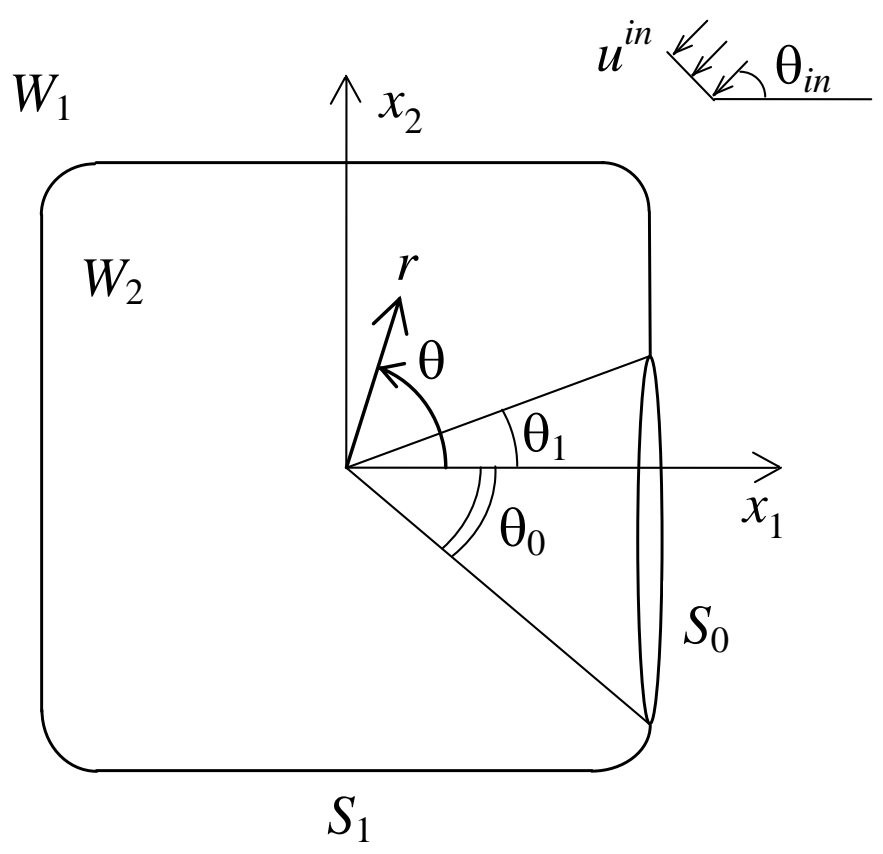

Fig.1. Geometry of the scattering problem.
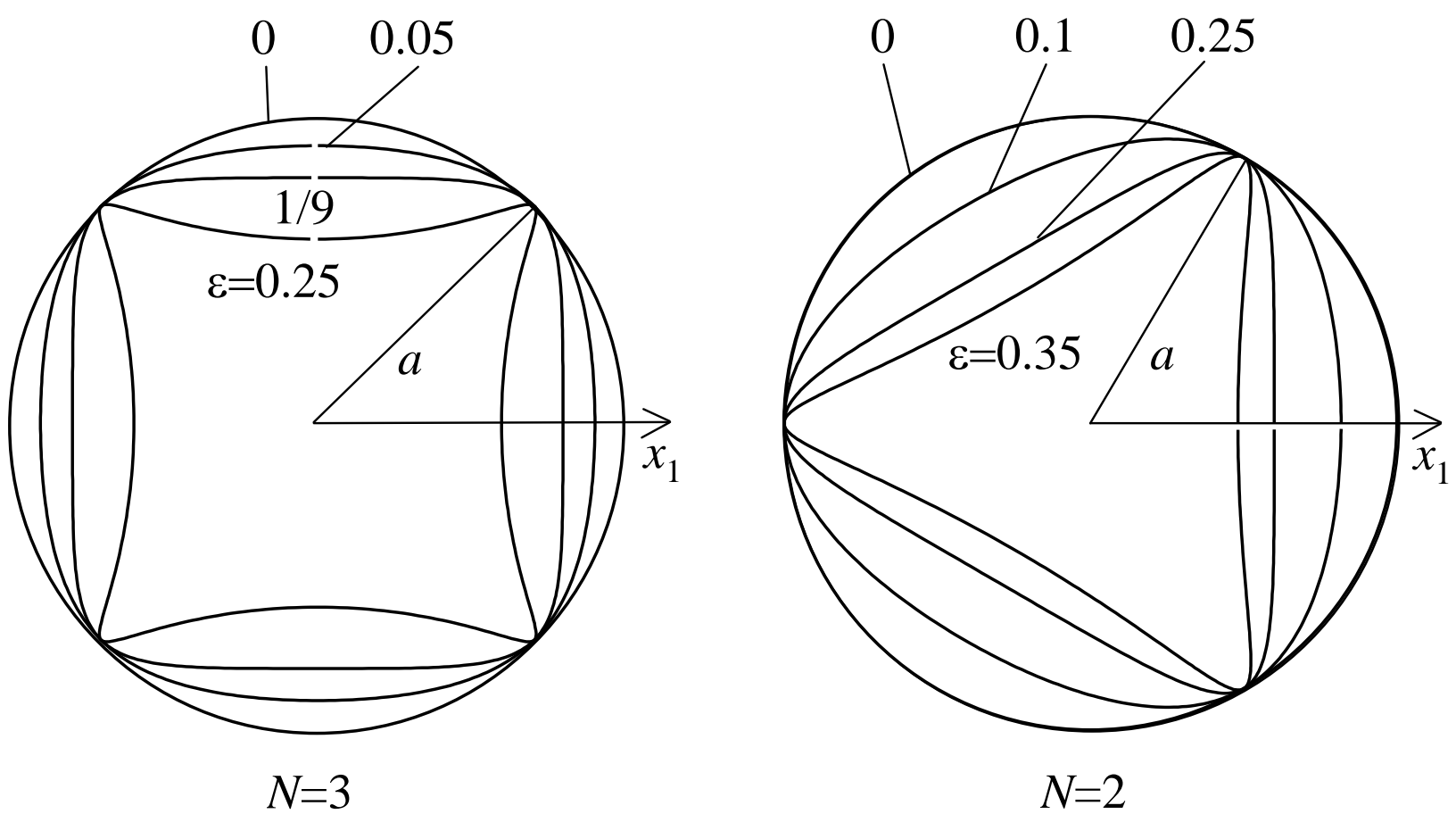

Fig. 2. Geometry of the fiber cross-section. 

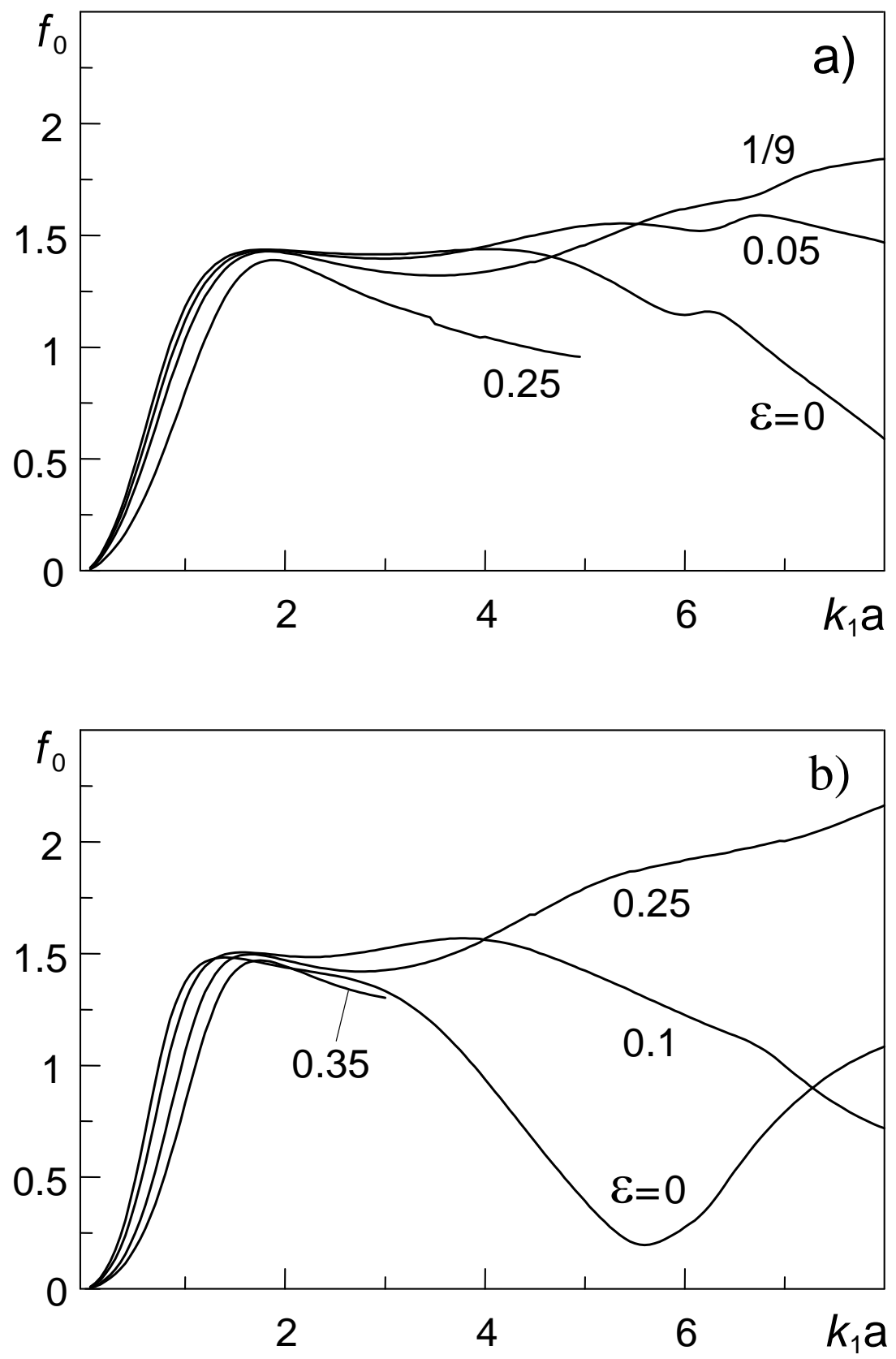

Fig. 3. The dimensionless backscattering amplitude for glass/epoxy composite with interface crack and different inclusion shapes $\left(\theta_{i n}=\theta_{s c}=0^{\circ}\right)$ : a) $\left.-N=3 ; \mathrm{b}\right)-N=2$. 

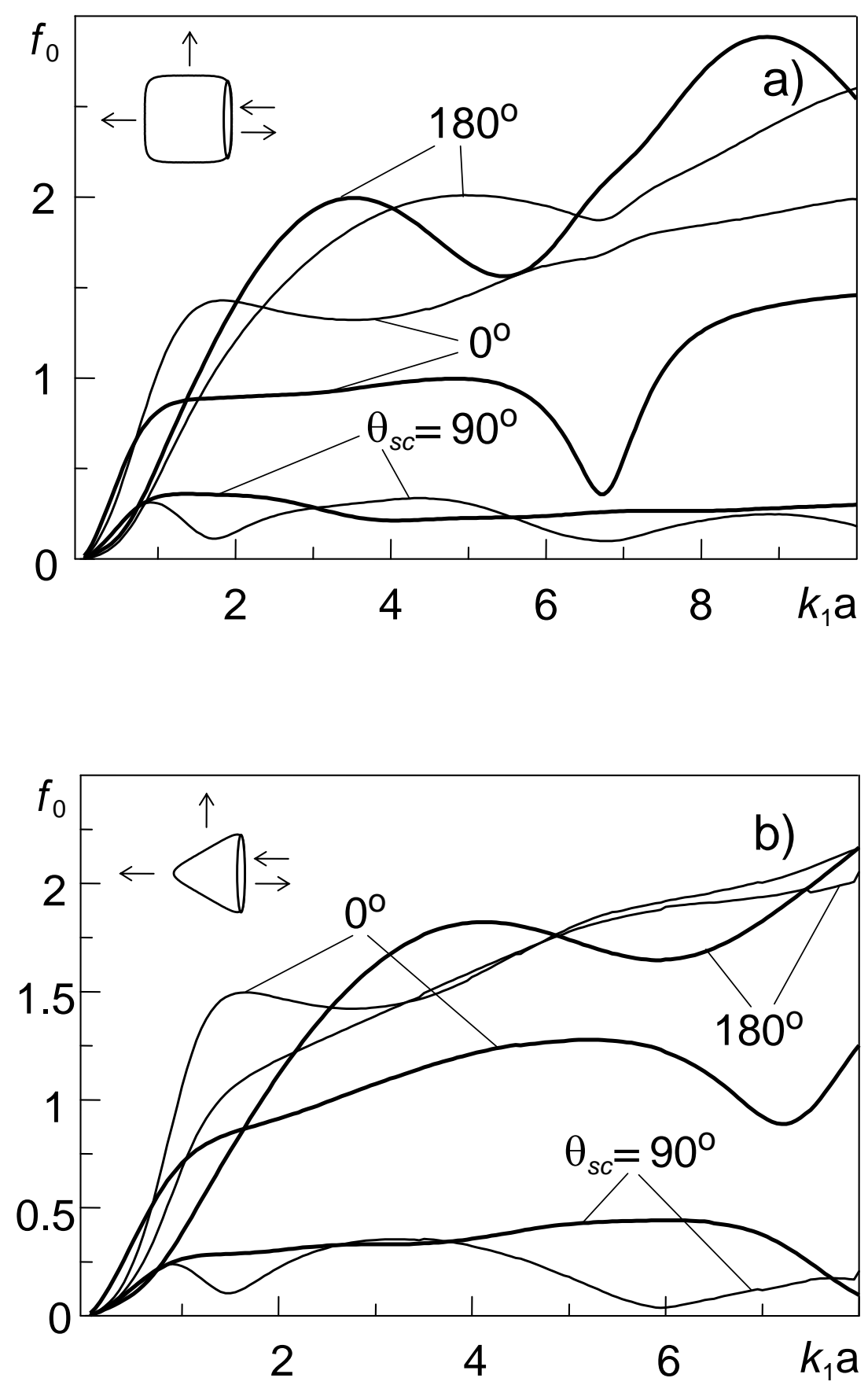

Fig. 4. The dimensionless scattering amplitude for glass/epoxy composite with and without (bold lines) interface crack and different angles of observation $\left(\theta_{i n}=0^{\circ}\right)$ :

a) $-N=3, \varepsilon=1 / 9 ;$ b) $-N=2, \varepsilon=0.25$. 

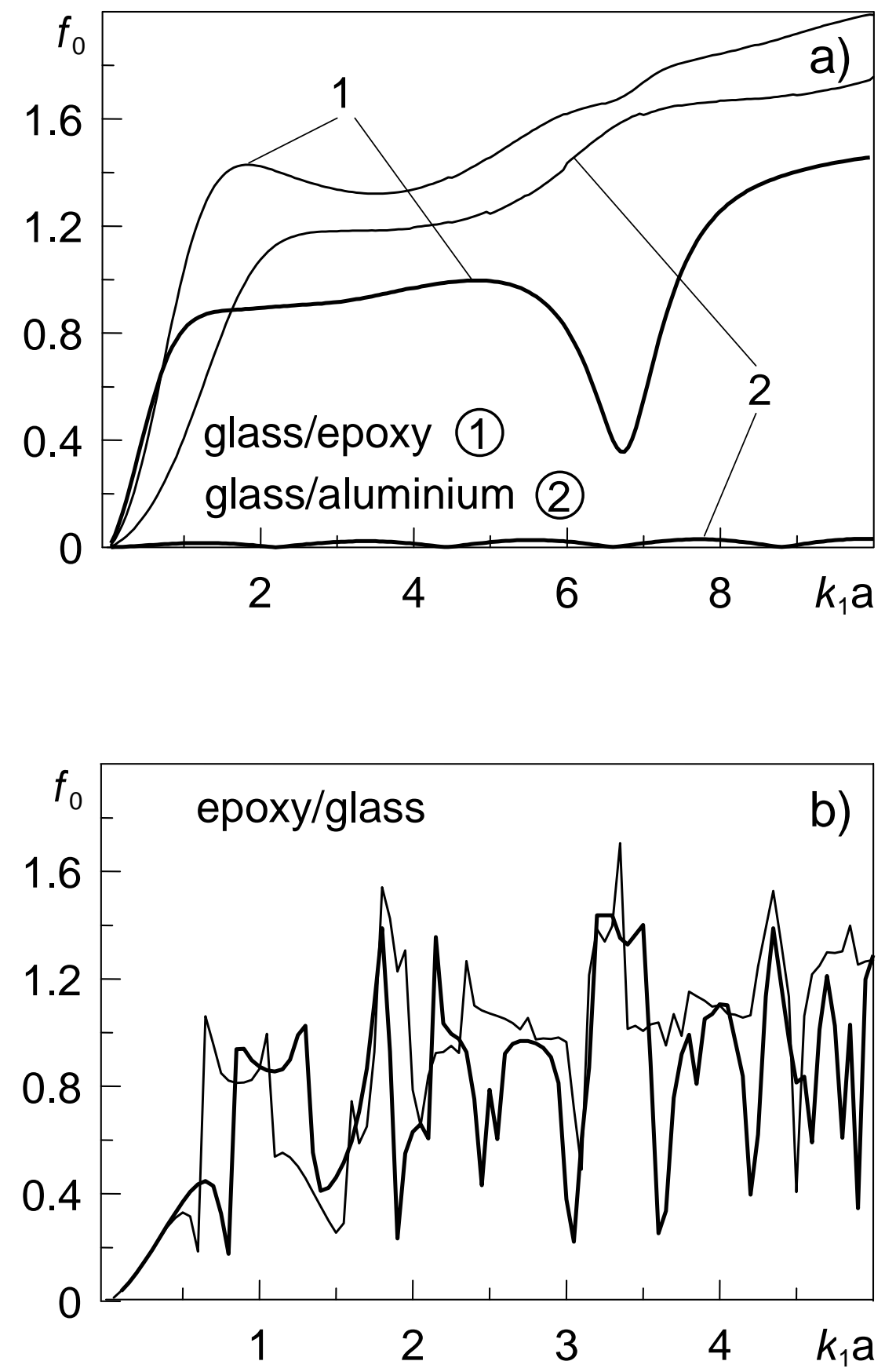

Fig. 5. The dimensionless backscattering amplitude for the quasi-square inclusion with and without (bold lines) interface crack and different matrix/inclusion materials $\left(\theta_{i n}=\theta_{s c}=0^{\circ}\right)$,

$$
N=3, \varepsilon=1 / 9 \text {. }
$$



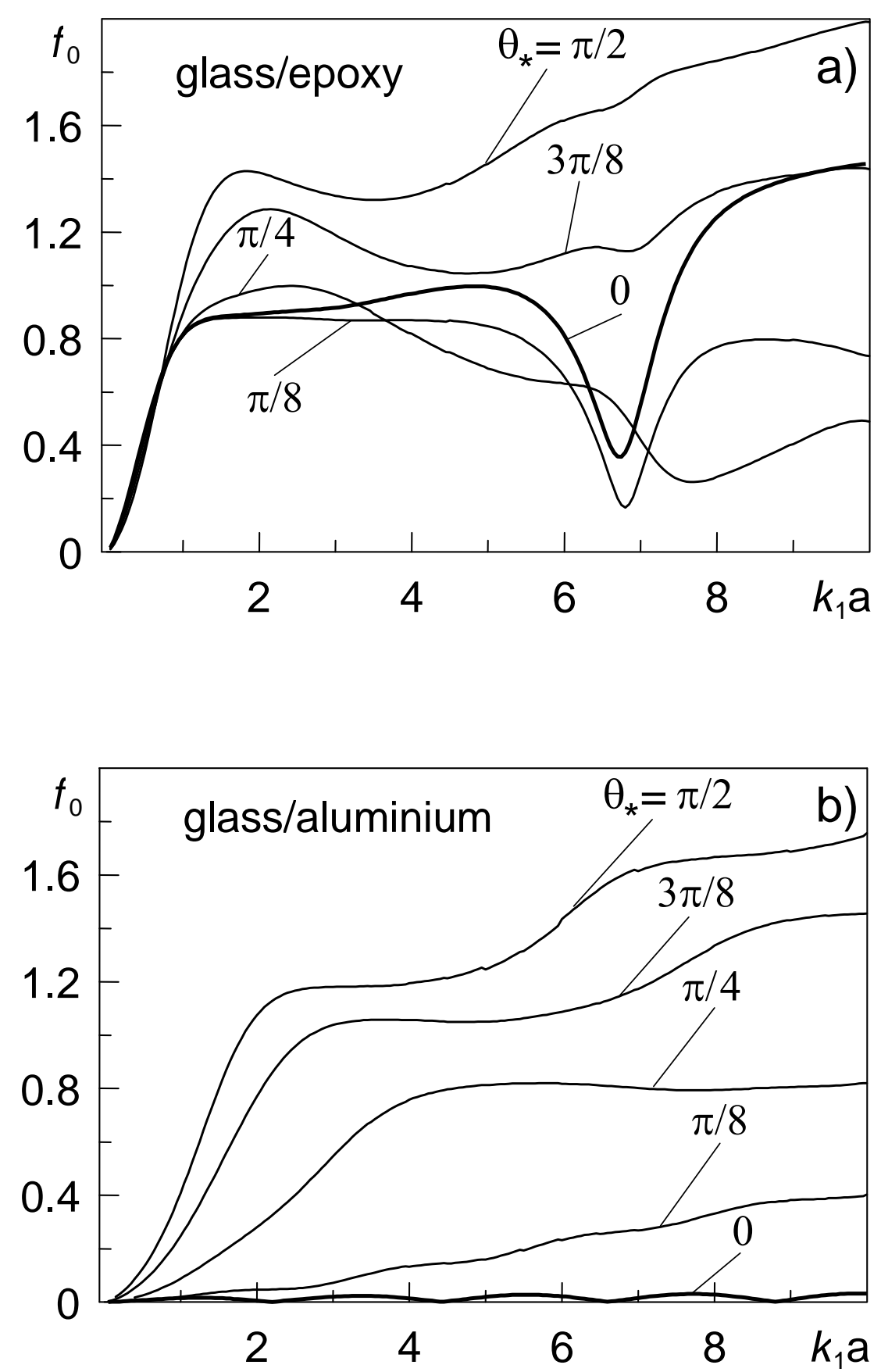

Fig. 6. The dimensionless backscattering amplitude for the quasi-square inclusion with and without (bold lines) interface crack and different crack sizes $\left(\theta_{i n}=\theta_{s c}=0^{\circ}\right), N=3, \varepsilon=1 / 9$. 


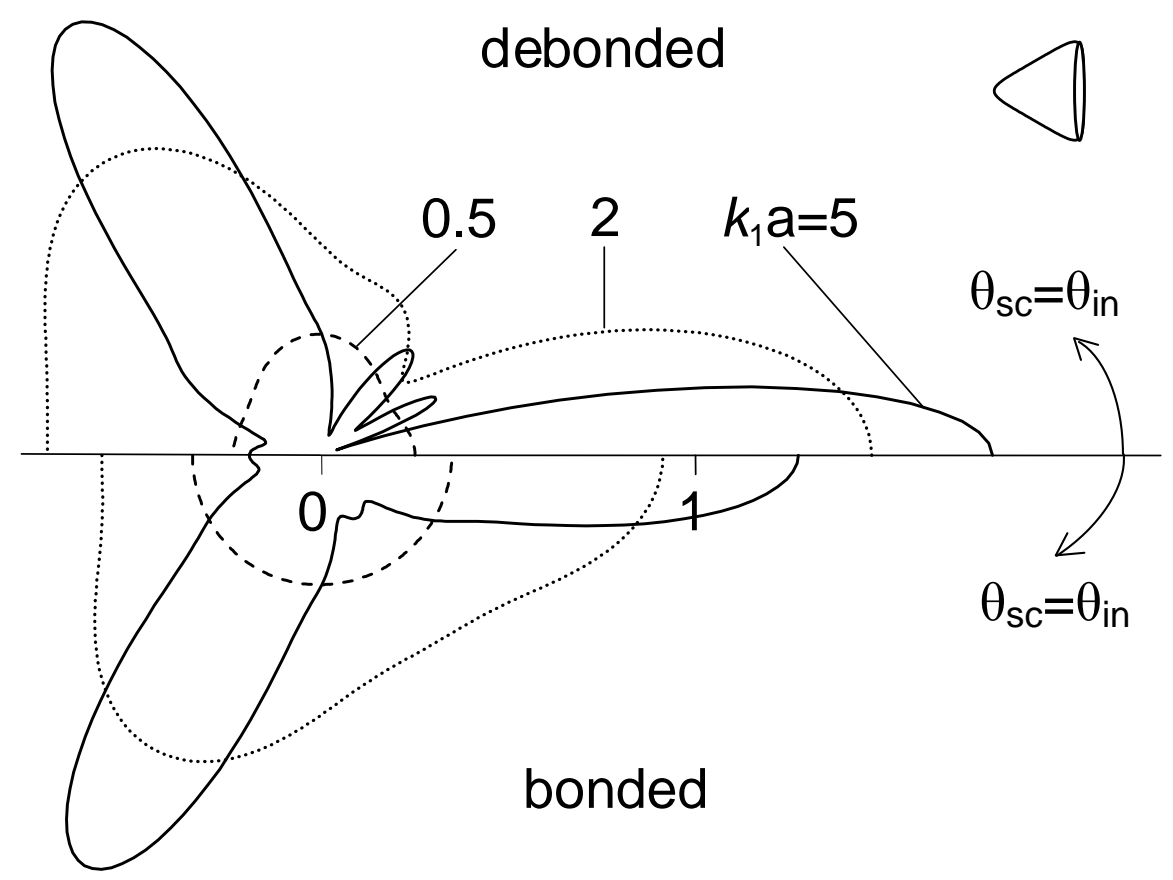

Fig. 7. The far-field pattern for glass/epoxy composite with and without interface crack for monostatic scattering $\left(\theta_{s c}=\theta_{i n}\right)$ and different wave numbers, $N=2, \varepsilon=0.25$. 